\title{
A Fokker-Planck Operator for the Emission and Absorption of Electron Plasma Waves in a Magnetized Plasma
}

\author{
Alan A. Ware \\ Institute for Fusion Studies \\ The University of Texas at Austin \\ Austin, Texas 78712
}

DOE/ET/53088--590

DE93 009579

\begin{abstract}
For slab geometry the perturbation of the electrostatic wake of a superthermal test electron in a magnetized plasma $\left(\omega_{c e} \gg \omega_{p e}\right)$ due to moderate magnetic shear is determined. Allowing for the spherical symmetry of the surfaces of constant phase to the rear of the test electron, the "resonant" field electrons causing the damping of the wave in a magnetic surface at a distance $x$ from the test electron are those with parallel velocity $v_{\|}^{\prime}=v_{! !} \cos 3 / \cos (\beta+\gamma)$. Here 3 is the angle between the emitted ray and $\mathbf{B}(0), y$ is the angle between $\mathbf{B}(0)$ and $\mathbf{B}(x)$ and $v_{\|}$is the velocity of the test electron. As a result the damping in the WKB approximaion for the wave emission is a function of both the angle of emission and $\gamma$. A Fokker-Planck equation is derived for the rate of change of the electron distribution function $(f)$ due to the emission and absorption of the waves under these conditions. $f$ is assumed approximately Maxwellian for $v_{\|} \lesssim v_{T}$ but with an arbitrary tail for $v_{\|}>v_{T}$.
\end{abstract}




\section{Introduction}

The anomalous electron energy loss in tokamaks has been known for more than two decades but has never been understood. In particular, the favorable scaling of energy containment with density and the square of the major radius for ohmically heated discharges has not been explained. In reversed-field pinch discharges there is not only anomalous electron energy loss but, as discussed in the preceding paper, ${ }^{1}$ there is diagnostic evidence for a strong transport of energy and momentum for the superthermal electrons associated with the current and for a strong interaction between such electrons. As pointed out there, analogous rapid transport of momentum and energy and strong particle interaction were observed for superthermal electrons in unrelated computer simulations by Decyk et al. ${ }^{2}$ The transport and interaction Were explained by Decyk th al. as being the result of electron plasma waves. (Anomalous particle transport was precluded by the nature of the simulation.)

Such waves exist even in a stable plasma, being emitted by electrons with velocity greater than the thermal velocity. Rostoker and Rosenbluth ${ }^{3}$ have shown that in thermodynamic equilibrium the energy level of modes with wavelengths longer than the Debye length is given by the classical Rayleigh-Jeans formula. The extra energy transport is given by the Poynting vector. For the case of an electrostatic mode Decyk, ${ }^{4}$ Amendt and Rostoker ${ }^{5}$ and others have shown that the divergence of the Poynting vector can be written in the form

$$
\boldsymbol{\nabla} \cdot \frac{c}{4 \pi} \mathbf{E} \times \mathbf{B}=\nabla \cdot \frac{\tilde{\Phi}}{4 \pi} \frac{\partial \widetilde{\mathbf{D}}}{\partial t},
$$

where $\widetilde{\Phi}$ and $\widetilde{\mathbf{D}}$ are the potential and dielectric displacement of the mode. Equation (1) indicates that high frequency waves are the importa $t$ ones. Electron plasma waves have such high frequency since for a magnetized plasma $\left(\omega_{c e} \gg \omega_{p e}\right)$ the mode frequency is given 
by

$$
\omega_{k}=\left(\frac{k_{\|}}{k}\right) \omega_{p e}\left(1+\frac{3}{2} k^{2} \lambda_{D}^{2}\right)
$$

with $\omega_{p e}$ in the range $10^{5}$ to $10^{6}$ megacycles for typical tokamak plasma. (upe. we are the electron plasma and cyclotron frequencies. respectively, $k_{\|}$is the component parallel to the magnetic field of the wave rector $\mathrm{k}$ and $\lambda_{D}$ is the electron Debye length $v_{T} / \sqrt{2} \omega_{p e}$.) These waves are reflected at the edge of the plasma where the density falls below the critical value for each particular mode.

The energy transport due to such electron plasma waves was calculated by Rosenbluth and Liu." They took a slab geometry model with uniform magnetic field. Assuming a sufficiently weak temperature gradient so that the local mode amplitudes are given by local thermodynamic equilibrium. a wave energy transport equation was used to determine the energy loss. Their result for the energy loss per unit volume from the center of the slab can be written in the form

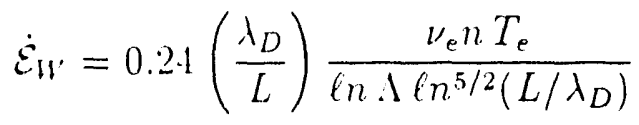

Where $L$ is the scale length for the temperature gradient and $\nu_{e}$ is the standard electron collision frequency $16 \sqrt{\pi} n \epsilon^{4}\left(n \Lambda / 3 m_{e}^{3} v_{T e}^{3}\right.$. Comparing this rate with the energy loss $\left(\dot{\mathcal{E}}_{\mathrm{c}}\right)$ due to classical electron heat conduction gave the ratio

$$
\frac{\dot{\varepsilon}_{W}}{\dot{\varepsilon}_{c}}=5 \times 10^{-2}\left(\frac{L}{\lambda_{D}}\right)\left(\frac{\lambda_{D}}{\rho_{e}}\right)^{2} \frac{1}{\ln \Lambda \ell n^{5 / 2}\left(L / \lambda_{D}\right)}
$$

where $\rho_{e}$ is the electron Larmor radius. Depending on the magnitudes taken for the various parameter this ratio lies in the range 1 to 10 , so that the loss due to the waves lies between the classical and the neoclassical electron heat conduction values, too small to explain the electron heat loss from toroidal plasmas. Rosenbluth and Liu were aware that the wave transport rate would be increased if the electron distribution function had an enhanced non-Maxwellian tail, but their method of calculation will not give the increase. 
The above analysis assumed that the waves involved are nomal modes. The modes with wave rector magnitude $k$ are entuded be electrons with relocity parallen to $\mathrm{B}$ given by rll such that

$$
k^{2} \lambda_{D}^{2} \simeq \frac{v^{2} T_{f}}{2 v^{2}}+\frac{3 v_{T}^{4}}{4 v_{t}^{4}}
$$

for $v_{\|}>v_{T e}$. The waves are damped by Landau damping with the resonant electrons having velocities centered about the same '"ll. It is the purpose of this paper to show that this damping is substantially changed when allowance is made for both the coherence of phase over the wake for each emitting electron and the effect of magnetic shear. In Sec. II, using the result of the preceding paper for the electrostatic wake of a superthermal test electron, the part of the wake corresponding to the emission of waves is identified. Section III gives an arproximate calculation for the damping of the waves allowing for the coherence of phase over the hemispherical wave fronts and the presence of magnetic shear. Only moderate magnetic shear is considered so that its effect on the wake can be treated as a perturbation to the uniform magnetic field case. This yields a WKB-type approximation for the damping of the wave which is a function of the angle of propagation and the angular rotation of the magnetic field due to the shear. From the results of Secs. II and III a Forker-Planck type operator is derived in Sec. IV for the rate of change of the electron distribution function due to the emission and absorption of the plasma waves for the above conditions.

\section{The Emitted Wave Packet}

In Ref. 1 the wake field was obtained for a superthermal electron in a uniform magnetic field for a magnetized plasma $\left(\omega_{c e} \gg \omega_{p e}\right)$. In the frame of reference moving with the test electron,

the $z$-axis being parallel to both $\mathbf{B}$ and the electron's parallel velocity $v_{\|}$, the potential in cylindrical coordinates is 
(a) for $z<0$

$$
\Phi(r, z)=\Phi_{0}-\Phi_{1}=-\frac{2 t}{R} \operatorname{cxp}\left(\frac{R}{\lambda_{1 \cdot f f}}\right) \cos k_{1} R-\Phi_{1}
$$

and (b) for $z>0$

$$
\Phi(r, z)=\Phi_{1}
$$

with $R=\left(r^{2}+z^{2}\right)^{1 / 2}$. $\Phi_{1}$ changes slowly from the value at $z=0$, namely

$$
\Phi_{1}(r, z=0)=-\frac{\epsilon}{r} \exp \left(-\frac{r}{\lambda_{\text {eff }}}\right) \cos k r,
$$

to the value for large $z$ given by

$$
\Phi_{1}(r, z)=-\frac{e}{z} \exp \left(-\frac{r}{\lambda_{\text {eff }}}\right)\left[J_{0}(k r)+\frac{1}{k \lambda_{\text {eff }}} N_{0}(k r)\right] .
$$

The general expressions for $k$ and $\lambda_{\text {eff }}$ are given in Ref. 1 . Here the electron distribution function $f$ is assumed to be approximately Maxwellian for $v \lesssim v_{T}$ and arbitrary for $v>$ 'T, except that the departure from Maxwellian is assumed sufficiently small so as not to significantly change the real part of the plasma dispersion function. Then

$$
\left.\begin{array}{l}
k \simeq \frac{\omega_{p}}{v_{\|}}=\frac{1}{\sqrt{2} \lambda_{D} x_{\|}} \\
\lambda_{\text {eff }}=\frac{-k \cdot m}{2 \pi^{2} e^{2}\left(\partial F / \partial v_{\|}\right)}
\end{array}\right\}
$$

witl: $x_{\|} \equiv v_{\|} / v_{T}$ and $F=\int_{0}^{x} 2 \pi v_{\perp} d v_{\perp} f$.

Intuitively it is $\Phi_{0}$, the first part of Eq. (5), which is to be identified as the emitted wave packet. Thus the resonance associated with the dispersion functiun gives a constant $k$ independent of the direction of $\mathrm{k}$. This is satisfied by $\Phi_{0}$. Secondly, the group velocity of these plasma waves is

$$
\mathrm{v}_{y}=\frac{\omega_{p} k_{z}}{k^{2}}\left(\frac{k_{x}}{k} \mathrm{i}_{x}+\frac{k_{y}}{k} \mathrm{i}_{y}+\frac{k^{2}-k_{z}^{2}}{k k_{z}} \mathrm{i}_{z}\right)
$$

in the stationary frame and hence in the frame of the test electron the group velocity is

$$
\mathrm{v}_{y}^{\prime}=\mathrm{v}_{y}-\iota^{\prime} \| \mathrm{i}_{z}=\frac{\omega_{p}^{\prime} k_{z}}{k^{2}}\left[\frac{k_{x}}{k} \mathrm{i}_{x}+\frac{k_{y}}{k} \mathrm{i}_{y}-\frac{k_{z}}{k} \mathrm{i}_{z}\right],
$$


since $c^{\prime} \|=\omega_{p} / k, v_{y}^{\prime}$ is therefore directed along the rarbii of the hemisphere to the rear of the test electron in this frame.

In addition the drag fore on the test electron due to the resonance is exactly enual to tike integral of the acceleration force on the resonant electrons causing the clamping in $\Phi_{1}$. The former was obtained by Rosenbluth and Rostoker but the derivation is reproduced here. not only because that report is not easily available but because this drag force will be an essential part of the Folker-Planck equation to be obtained in Sec. IV.

Starting from $E_{1}$. (3) of Ref. I the force on the test electron is

$$
\begin{aligned}
& F_{\|}=\left.\epsilon \frac{\partial \Phi}{\partial z}\right|_{\substack{i=0 \\
z=0}}=\text { Real Part } \frac{-\epsilon^{2} \lambda_{D}^{2}}{2 \pi^{2}} \int d^{3} k\left[\frac{i k_{z}}{h^{2} \lambda_{D}^{2}-W_{R}+i W_{l}\left(k_{z} /\left|k_{z}\right|\right)}\right] \\
& =-\frac{-\epsilon^{2} \lambda_{D}^{2}}{2 \pi^{2}} \int k^{3} d k \int_{0}^{2 \pi} d \phi \int_{0}^{\pi / 2} \frac{2 W_{l} \cos \theta \sin \theta d \theta}{\left(k^{2} \lambda_{D}^{2}-W_{R}\right)^{2}+W_{I}^{2}} \\
& =-\frac{\epsilon^{2}}{4 \pi \lambda_{D}^{2}} \int d k\left[\frac{4 k \lambda_{D}^{2}\left(k^{2} \lambda_{D}^{2}-W_{R}\right) W_{I}+4 k \lambda_{D}^{2} W_{R} W_{I}}{\left(k^{2} \lambda_{D}^{2}-W_{R}\right)^{2}+W_{I}^{2}}\right] \\
& =-\frac{2 n \epsilon^{4} W_{l}}{m v_{T}^{2}} \mid\left(\left.n\left[\left(k^{2} \lambda_{D}^{2}-W_{R}^{2}\right)^{2}+W_{I}^{2}\right]\right|_{k_{\min }} ^{k_{\max }}-\frac{4 n e^{4} W_{R}}{m v_{T}^{2}}\left|\tan ^{-1}\left(\frac{k^{2} \lambda_{D}^{2}-W_{R}}{W_{I}}\right)\right|_{k_{\min }}^{k_{\max }} \cdot(12)\right.
\end{aligned}
$$

$k_{\text {min }}$, the minimum value of $k$, will be $\rho_{e}$ for fusion plasmas since the magnetized plasma approximation which neglects the perpendicular motion of the electrons ceases to be valid for collision parameters less than $\rho_{e}$. $k_{\max }$ will be $\lambda_{\text {eff }}^{-1}$. The second term is that associated with the resonance giving wave emission. Provided $\lambda_{D}^{2} \rho_{e}^{-2} \gg W_{R} \gg W_{l}^{\prime}$, this term is given approximately by

$$
F_{\| W}=-\frac{2 \pi n e^{4}}{m v_{\|}^{2}},
$$

for a Maxwellian plasma. This is the result obtained by Rosenbluth and Rostoker.

The first part of Eq. (12) is a contribution to the frictional drag due to collisions with the field electrons. It is incomplete because the analysis treats the background electrons as a continuous fluid and there is no allowance being made for the recoil of the test electron due to each collision. 
Tuming to the force on the resonant fiedel electrons, the dhatge density associated with the potential $\Phi_{0}$ for $z<0$ is

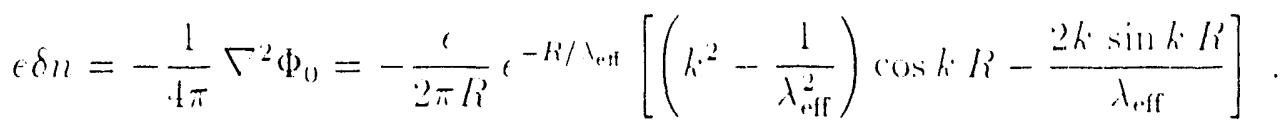

The resonant part of this density is that proportional to sin $k R$, being in phase with the wave part of the electric field whose z-component is

$$
E_{z}=\frac{2 e}{R} e^{R / \lambda_{n+\pi}} k \cos \theta \sin k R
$$

where $\cos \theta=|z| / R$. Hence the total force on the resonant electrons is

$$
\begin{aligned}
F_{\|} & =\int d^{3} r e \delta n E_{z} \\
& =\int_{0}^{\infty} 2 \pi R^{2} d R \int_{0}^{\pi / 2} \cos \theta \sin \theta d \theta\left(\frac{2 e^{2} k^{2}}{\pi R^{2} \lambda_{\mathrm{eff}}} e^{-2 R / \lambda_{\mathrm{eff}}} \sin ^{2} k R\right) \\
& =\frac{\epsilon^{2} k^{2}}{2} \int_{0}^{x} \frac{2}{\lambda_{\mathrm{eff}}} \epsilon^{-2 R / \lambda_{\mathrm{eff}}} d R=\frac{2 \pi n e^{4}}{m v_{\|}^{2}}
\end{aligned}
$$

for $k: \lambda_{\text {eff }}^{-1}$ and $k^{2} \lambda_{D}^{2} \simeq 1 / 2 x_{\|}^{2}$. This gives the result that the force on the resonant field electrons exactly balances the drag force on the test electron due to wave emission given in $\mathrm{Eq} \cdot(13)$.

For the reasons given in this section, the potential $\Phi_{0}$ is taken to be the wave packet associated with ware emission by the test electron and the part $\Phi_{1}$ is neglected. (The only large electric fields associated with $\Phi_{1}$, namely of order $k \Phi_{1}$, are directed parallel to the cylindrical radius vector $\mathbf{r}$, normal to $\mathbf{B}$. Estimates indicate that little energy is given to field electrons and $\Phi_{1}$ will not contribute significantly to energy transport. However, these fields could enhance electron self-diffusion of the type studied by O'Neil. ${ }^{8}$ )

\section{The Effect of Magnetic Shear}

Rosenbluth and Liu showed that the most important waves for energy transport are those with Landau damping mean free path, $\lambda_{\text {eff }}$, equai to the scale length for the electron distri- 
bution function at the corresponding value of "wh whe was $L_{T} / x^{\prime}$ in their model where $L_{T}$ is the scale length for the temperature gradient. This gives $\lambda_{\text {eff }}=L_{T} / x_{\|}$and the optimm value of $x_{\| 1}^{2}$ is

$$
r_{m}^{2}=\left(n\left[\left(\frac{\pi}{2}\right)^{1 / 2} \frac{x_{m} L_{T}}{\lambda_{D}}\right] \simeq \ln \left(\frac{L_{T}}{\lambda_{D}}\right) .\right.
$$

For lower values of $x_{\|}$, $\lambda_{e f f}$ decreases exponentially giving less transport because of the smaller step length. For larger values the damping of the waves saturates, due to the rapidly decreasing number of resonant particles with distance and reflection of the waves brings some of the energy back to the central region.

A typical value of $x_{m}^{2}$ is $s$ and Rosenbluth and Liu neglected the small variations in $k$ over the limited length $L_{T} / x_{m}$ and used a WKB approximation to allow for the stronger variation in $\lambda_{\text {eff }}$. Essentially in $\Phi_{0},\left(E_{q} .(5)\right)$ the factor $\exp \left(-R / \lambda_{\text {eff }}\right)$ was replaced by

$$
\exp \left[-\left(\frac{\pi^{1 / 2} x_{m}^{2}}{\sqrt{2} \lambda_{D}}\right) \int_{0}^{R} e^{-m v^{2} / 2 T(R)} d R\right]
$$

where the relation $k=\omega_{p} / v^{\prime}$ has been used. Our concern here is to obtain the corresponding WKB approximation when allowance is made for magnetic shear and the wave coherence over each hemisphere in $\Phi_{0}$. It is necessary to allow for the weak variation of $\mathrm{k}$ to avoid the Fokker-Planck equation giving a fictitious transport of energy due to either a density gradient or magnetic shear when the electron distribution is Maxwellian and there is uniform temperature. The calculation will be performed to first order, in $\delta k / k_{0}$, where $\delta k$ is the change in $k$.

For simplicity only slab geometry is considered with $O z$ again parallel to $\mathbf{B}$ at the position of the test electron and moving with the test electron. $O y$ is the other axis in the magnetic surface and $O x$ is the direction in which the quantities $n, T_{e}$, and the direction of $\mathbf{B}$ vary. The parallel velocity of the test electron is now denoted by $v_{0}$ and $v_{0} / v_{T}$ by $x_{0}$, with $v_{\|}, x_{\|}$ used for a field electron. A ray emitted by the test electron in a particular direction will have a curved path due to the change in $\mathrm{k}$ with $x$. At a distance $s$ along the ray the local 
dispersion relation for large $x_{0}$ is

$$
k_{s}=\frac{k_{i n s} \omega_{i p s}}{\omega}=\frac{k_{1 y=} \cos (. j+i) \mu_{i p s}}{\omega} .
$$

Here $k_{y z}=\left(k_{y}^{2}+k_{z}^{2}\right)^{1 / 2}=h_{s}$ cos $p_{s}$. Which remanis unchanged along the rays 3 is the angle between the projection of the ray on the $y=$ plane and the z-axis and $q$ is the small angle through which $\mathbf{B}$ has rotated in the distance $x .\left[\sin y=\sin \left(x / L_{s}\right) \simeq x / L_{s}\right.$ where $L_{s}$ is the shear length.] Only weak shear is considered $\left(L_{s} \gg L_{T} / x_{0}\right)$ so that the change from uniform $\mathbf{B}$ can be treated as a perturbation. For the particular ray at $\mathbf{R}=0$

$$
\omega^{\prime}=\frac{k_{y z} \cos 3 \omega_{p 0}}{k_{v}}
$$

so that

$$
k_{s}=\frac{\cos (\beta+\gamma)}{\cos \beta}\left(\frac{n_{s}}{n_{0}}\right)^{1 / 2} k_{0}
$$

At the point s the group relocity from $\mathrm{Eq}_{\mathrm{q}}$ (10) in the moving frame of the test electron is

$$
\mathrm{v}_{y s}=\left(\frac{\mathrm{k}_{s}}{k_{s}}\right) \frac{k_{\| s} \omega_{p s}}{k_{s}^{2}}+\frac{\omega_{p s}}{k_{s}} \mathrm{i}_{\|}-v_{0} \mathrm{i}_{z}
$$

with $\mathbf{i}_{\|}=\mathbf{i}_{z} \cos \gamma+\mathbf{i}_{y} \sin \gamma$. The last two torms in Eq. (19) have the magnitude $\Delta v_{y}=$ $v_{0} \sin \gamma / \cos (\beta+\gamma)$ and the direction of $\Delta \mathbf{v}_{g}$ is perpendicular to the projection of $\mathbf{k}_{s}$ on the yz-plane. Since, is taken to be small, $\Delta \mathbf{v}_{y}$ will be neglected with respect to the first term on the right of $\mathrm{Eq} .(19)$. Large values of $\beta$ approaching $\pm \pi / 2$ are not important because little energy is transmitted in these directions, $v_{y}$ being small, and because the forces on the test electron and field electrons are proportional to $\cos \beta$ and $\cos (\beta+\gamma)$. Then $\mathbf{v}_{g s}$ is parallel to $\mathrm{k}_{s}$ and since $k_{y z}$ remains unchanged along a ray

$$
\cos \varphi_{s}=\frac{k_{0}}{k_{s}} \cos \varphi_{0}
$$

where $\varphi_{0}$ is the value of $\varphi_{s}$ at $R=0$. 
An element of solid angle near $\mathbf{R}=0$ is given by $\delta \Omega=\cos$ quosos 8.3 and the energy flowing out through als due to the wave emission from the test electron is

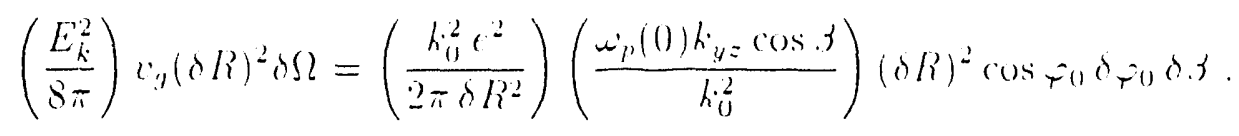

At this stage the damping is neglected and $\Phi_{0}$ is assumed to have the approximate form

$$
\Phi_{0}=-\frac{2 C \epsilon}{R} \cos \left(\int_{0}^{s} k(s) d s\right) .
$$

where $C$ is the WKB amplitude factor to allow for the variation of $\mathrm{k}$ and is the first unknown to be determined. At a distance $s$ along the ray where the position vector is $\mathbf{R}$, the area subtended by $\delta \Omega$ is $\left(R \cos \varphi_{m} \delta \beta\right) \delta x$, where

$$
R \cos \varphi_{m}=\int_{u}^{s} \cos \varphi_{s} d s \simeq R \cos \varphi_{0}\left(1-\frac{\bar{\delta} k}{k_{0}}\right)
$$

with $\bar{\delta} k=s^{-1} \int_{0}^{s} \delta k: d s$.

$$
\delta_{r_{\nu}}=\int_{0}^{s}\left(\delta_{\varphi_{0}}+\int_{0}^{s} \frac{\partial\left(\delta_{\varphi_{s}}\right) d s}{\partial s}\right) d s .
$$

where $\delta \varphi_{s}$ is the angular spread at s corresponding to $\delta \varphi_{0}$. From Eq. (20)

$$
\frac{\partial_{\varphi_{s}}}{\partial s}=\frac{k_{0}}{k_{s}^{2}} \frac{\cos \varphi_{0}}{\sin \varphi_{s}} \frac{\partial(\delta k)}{\partial s} \simeq \frac{\cos \varphi_{0}}{k_{0}} \frac{\partial(\delta k)}{\partial x}
$$

to first order in $\delta k$ and

$$
\frac{\partial\left(\varphi_{s}+\delta \varphi_{s}\right)}{\partial s}=\frac{\cos \left(\varphi_{0}+\delta \hat{\varphi}_{0}\right)}{k_{0}} \frac{\partial(\delta k)}{\partial x}
$$

so that

$$
\frac{\partial}{\partial s}\left(\delta_{\varphi_{s}}\right) \simeq-\frac{\sin \varphi_{0}}{k_{0}} \frac{\partial(\delta k)}{\partial x} \delta_{\varphi_{0}} \simeq-\frac{\delta \varphi_{0}}{k_{0}} \frac{\partial(\delta k)}{\partial s} .
$$

Substituting from Eq. (25) into Eq. (24)

$$
\delta x_{\nu}=s \delta_{\varphi_{0}}\left(1-\frac{\bar{\delta} h}{k_{0}}\right) \simeq R \delta \varphi_{0}\left(\frac{\cos \varphi_{m}}{\cos \varphi_{0}}\right)
$$


since to first order in $\delta k, s=R$. Hence the energy flowing throngh the element of area at $s$ is

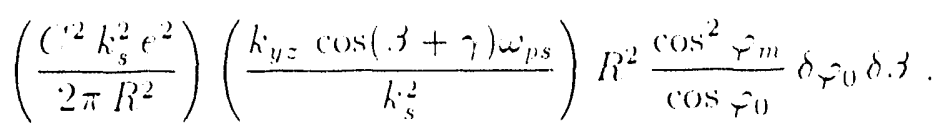

Equating this energy flow with that at $\delta R$

$$
C^{2}=\frac{h_{0}}{h_{s}} \frac{\cos ^{2} \hat{\varphi}_{0}}{\cos ^{2} \hat{\varphi}_{m}}
$$

using Eq. (18).

Introducing an unknown WKB type damping into Eq. (22), namely

$$
\Phi_{0}=-\frac{2(' t}{R} \exp \left(-\int_{0}^{s} \mu_{s} d s\right) \cos \left(\int_{0}^{s} k_{s} d s\right) \text {, }
$$

the energy flow at the point $\mathbf{R}$ now has a divergence given by

$$
\frac{1}{R^{2} \cos ^{2} \varphi_{m}} \frac{\partial\left(v_{g} \frac{E^{2}}{8 \pi} R^{2} \cos ^{2} \varphi_{m}\right)}{\partial s}=-2 \mu_{s} v_{g} \frac{E^{2}}{8 \pi} \text {. }
$$

To determine $\mu_{s}$ the field particle orbits will be used to determine $\delta n_{s}$, the part of $\delta n$ in phase with the wave electric field and the rate at which energy is given to these particles will be equated to Eq. (29).

Figure 1 represents the magnetic surface at the distance $x$ from the test electron and the line marked $u$ is the unperturbed orbit of a field electron whose velocity in the moving frame is

$$
\mathbf{u}=\left(v_{\|} \cos \gamma-v_{0}\right) \mathbf{i}_{z}+v_{\|} \sin \gamma \mathbf{i}_{y},
$$

$\mathrm{v}_{\|}$being the electron's parallel velocity in the rest frame. If $P$, the point of closest approach, is a distance $R$ from the test electron

$$
O P=R_{n} \cos \hat{\varphi}_{m}
$$

The angle 3 between $O P$ and the $z$-axis is given by

$$
\tan \beta=\frac{\left(v_{\|} \cos \gamma-v_{0}\right)}{\iota^{\prime} \| \sin \gamma}
$$


or alternatively:

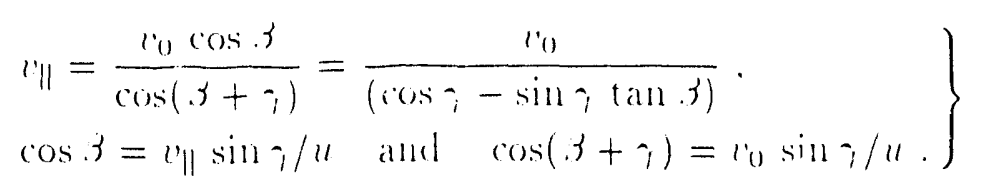

Considering initially the total potential $\Phi$, if the electric field at a point on the orbit is divided into the two components $\mathbf{E}_{\rho}, \mathbf{E}_{\perp}$, parallel and perpendicular to the orbit, the parallel momentum gained by the field electron for the complete orbit is

$$
m \Delta v_{\|}=\frac{-\epsilon}{u} \int_{-\infty}^{+\infty} \mathbf{i}_{\|} \cdot\left(\mathbf{E}_{\rho}+\mathbf{E}_{\perp}\right) d \ell
$$

where $\ell$ measures distance along the orbit from point $P$. Since $E_{\ell}=-\partial \Phi / \partial \ell$ the $\mathbf{E}_{\ell}$ part gives zero; only $\mathbf{E}_{\perp}$ gives a contribution. The wave part of $\mathbf{i}_{\|} \cdot \mathbf{E}_{\perp}$ at the point $\ell$ is

$$
\mathrm{i}_{\|} \cdot \mathbf{E}_{\perp}=\frac{2\left(\cdot \cos \left(\varphi_{s}-\delta_{\varphi}\right)\right.}{\left(R^{2}+\ell^{2}\right)^{1 / 2}} \frac{R \cos \varphi_{m} \cos (\beta+\gamma+\delta \beta)}{\left(R^{2} \cos ^{2} \varphi_{m}+\ell^{2}\right)^{1 / 2}} e^{-A_{\ell}} k_{s} \sin \left|\int_{0}^{s} k_{s} d s\right|_{\ell}
$$

where $-\delta \varphi, \delta \beta$ are the increments in $\varphi_{s}$ and $(\beta+\gamma)$ caused by the displacement $\ell$ from point $P$ and $A_{\ell}$ is the value of $\int_{0}^{s} \mu_{s} d s$ for the point $\ell$.

Of the various terms in Eq. (35) which are functions of $\ell$, the one which varies rapidly is the sine term. In the appendix it is shown that if the value of the phase $\int_{0}^{s} k_{s} d s$ at point $P$ is $\Theta$ then the phase at $\ell$ is

$$
\Theta+k_{0} \delta r \cos \varphi_{m}
$$

to first order in $\delta k$ and $\delta r$, where

$$
\delta r=O \ell-O P=\left(R^{2} \cos ^{2} \varphi_{m}+\ell^{2}\right)^{1 / 2}-R \cos \varphi_{m} \simeq \frac{\ell^{2}}{2 R \cos \varphi_{m}} .
$$

Using these results and substituting Eq. (35) into Eq. (34)

$$
\begin{aligned}
m \Delta v_{\|} & =-\frac{2\left(e^{2} k_{y z} \cos (\beta+\gamma)\right.}{u R} e^{-A_{p}} \int_{-\infty}^{+\infty} \sin \left(\Theta+\frac{k_{0} \ell^{2}}{2 R}\right) d \ell \\
& =-\frac{2 \sqrt{2} C e^{2}}{u}\left(\frac{\pi}{k_{0} R}\right)^{1 / 2} k_{y z} \cos (\beta+\gamma) e^{-A_{z}} \sin \left(\Theta+\frac{\pi}{4}\right)
\end{aligned}
$$


where for the other factors in Eq. (3.)) the value at $P$ have been taken. This approximation is justified since the illustration of the Fresnel integrals in Fig. 2 shows that the field electron gains the momeritum of Eq. (36) essentially orer the short distance $2 \Delta \ell$ where $k_{0}(\Delta \ell)^{2} / 2 R=$ $\pi / 2$ or $\Delta \ell=\left(\pi R / k_{u}\right)^{1 / 2}$. The net effect of the other terms depending on $\ell$ in $E_{1} .(35)$ is to reduce the amplitude of the oscillations at large $\ell$. The result Eq. (36) will be used in Sec. IV.

If in Eq. (36) the limits of integration are taken as $-\infty$ and 0 the $\Delta v_{\|}$is reduced by a half. (The integral for $\mathbf{E}_{\ell}$ in $\mathbf{E}_{\mathrm{q}}$. (34) is now nonzero, but since it is proportional to $\cos \Theta$ it is omitted.) In Fig. 1, considering the two orbits at angles $\pm \Delta \beta$ to the original orbit, the orbit for $+\Delta \beta$ will give half the result of $\mathrm{Eq}$. (36), with $O R$ replacing $R \cos \varphi_{m}$, less the integral from $P$ to $R$ and for $-\Delta, 3$ the integral is half $\mathrm{Eq}_{\mathrm{q}}$ (36), with $O Q$ replacing $R \cos \varphi_{m}$, plus the integral from $Q$ to $P$. The contribution of these two orbits to the perturbed density at $P$ is therefore

$$
\begin{gathered}
\left(d v^{\prime} \| \frac{\partial F}{\partial v_{\|}}\right) \frac{2 \sqrt{2} C e^{2}}{m u}\left(\frac{\pi}{k_{0} R_{R}}\right)^{1 / 2} e^{-A_{K}} k_{s} \cos (\varphi+\delta \varphi) \cos (\beta+\gamma) \\
\times \sin \left\{\Theta+\frac{\pi}{4}-k_{0} \cos \varphi_{m}\left[\frac{R \cos \varphi_{m}}{2}(\Delta \beta)^{2}\right]\right\},
\end{gathered}
$$

since $O R=O Q$ and $\left.\delta r=R \cos \varphi_{m}-O R=R \cos \varphi_{m}[1-\cos (\Delta \beta)]=R \cos \varphi_{m}(\Delta \beta)^{2}\right) / 2$. The approximation $\cos (\beta+\gamma+\Delta \beta)+\cos (\beta+\gamma-\Delta \beta)=2 \cos (\beta+\gamma)$ has been made and the integrals over $Q P$ and $P R$ have been taken equal. $F$ is the one-dimensional electron distribution function at the point $P, F=\int_{0}^{\infty} 2 \pi v_{\perp} d v_{\perp} f(\mathbf{R})$.

From Eqs. (32) and (33), $\Delta \beta=\Delta \imath^{\prime} \| \cos (3+\gamma) / u$ and hence

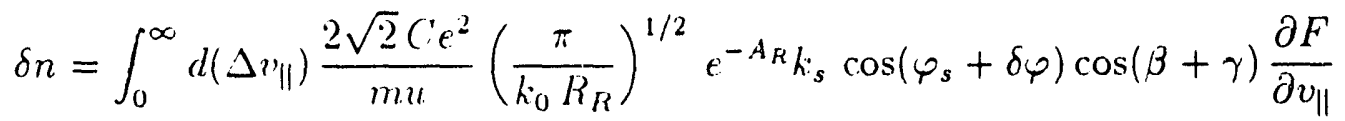

$$
\begin{aligned}
& \times \sin \left[\Theta+\frac{\pi}{4}-\frac{k_{0} R \cos ^{2} \varphi_{m} \cos ^{2}(\beta+\gamma)}{2 u^{2}}\left(\Delta v_{\|}\right)^{2}\right] \text {. }
\end{aligned}
$$


Once again it is the sine term which varies rapidly with $\Delta c^{\prime} \|$. Taking the value of the other factors at $\Delta v^{\prime}=0$.

$$
\delta_{n}=\frac{2 \pi C^{2} c^{2}}{m R} \frac{k_{y z}}{h_{u} \cos \varphi_{m}} e^{-A_{n}} \frac{\partial F}{\partial v_{\nu} \|} \sin \Theta,
$$

where $\partial F / \partial v^{\prime \prime \mid}$ is for the '\| given by Eq. (33), which gives the orbit perpendicular to $\mathrm{k}$ at the point $P$. Thus the "resonant." electrons causing the damping are centered about this value.

In the laboratory frame the work done on $\delta n$ due to the wave field, when averaged over a wavelength is

$$
\left\langle-v_{\|} \delta n e \mathbf{i}_{\|} \cdot \mathbf{E}_{k}\right\rangle=-\frac{2 \pi C^{2} e^{4}}{m R^{2}} \frac{k_{\|} k_{y z}}{k_{0} \cos \varphi_{m}} \exp \left(-2 A_{p}\right) v_{\|} \frac{\partial F}{\partial v_{\|}} .
$$

Equating Eqs. (29) and (39)

$$
\mu_{s}=-\frac{2 \pi^{2} e^{2}}{m} \frac{k_{y z}}{k_{0} \cos \varphi_{m}} \frac{v_{\|}}{\omega_{p}(s)} \frac{\partial F}{\partial v_{\|}}=-\frac{2 \pi^{2} e^{2}}{m} \frac{\cos \varphi_{0}}{k_{s} \cos \varphi_{m}} \frac{\partial F}{\partial v_{\|}}
$$

and the potential $\Phi_{0}$ for moderate shear is

$$
\Phi_{0}=\left(\frac{k_{s}}{k_{0}}\right)^{1 / 2}\left(\frac{\cos \varphi_{0}}{\cos \varphi_{m}}\right) \frac{2 \epsilon}{R} e^{-\int_{0}^{s} \mu, d_{s}} \cos \int_{0}^{s} k_{s} d s
$$

with $s \simeq R$ and $k_{s}, \mu_{s}$ and $\varphi_{m}$ are given by Eqs. (17), (40), and (23).

\section{The Fokker-Planck Operator}

Since only " $\|$ is affected by the emission and absorption of the plasma waves, the FokkerPlanck operator for the rate of change of $f$ due to the emission and absorption of the plasma waves is one-dimensional and has the form

$$
C_{W}(f)=-\frac{\partial}{\partial v}\left[\left\langle\Delta v_{\|}\right\rangle f-\frac{1}{2}\left\langle\left(\Delta v_{\|}\right)^{2}\right\rangle \frac{\partial f}{\partial v_{\|}}\right] .
$$

Here, $\left\langle\Delta v_{\|}\right\rangle$is the average change in $v_{\|}$per unit time due to wave emission and $\left\langle\left(\Delta v_{\|}\right)^{2}\right\rangle$ is the average of $\left(\Delta v_{\|}\right)^{2}$ over all the possible encounters of a test electron with the waveemissions of field electrons ahead of the test electron, times the number of such encounters 
per unit time. $\left\langle\Delta v^{\prime}\right\rangle$ has already been olitained in Eq. (12)

$$
\left\langle\Delta v_{\|\|}\right\rangle=\frac{F_{\| W}}{m}=-\frac{2 \pi n \epsilon^{4}}{m^{2} v_{\|}^{2}} \text {. }
$$

This formula is strictly accurate for the case of uniform magnetic field and density; since the shear and density gradient are assumed weak, it will be taken as good approximation here.

The value of $\Delta v^{\prime}$ for a field electron was obtained in $\mathrm{Eq} .(36)$ for a single encounter and the corresponding value for the test electron interacting with the wave emission of a single field electron is

$$
\Delta v_{\|}=\frac{2 \sqrt{2} C^{\prime} \epsilon^{2}}{m u} k_{y z}\left(\frac{\pi}{k_{p} l}\right)^{1 / 2} \cos (\pi+\beta) e^{-A} \sin \left(\Theta+\frac{\pi}{4}\right),
$$

where $p$ is now taken as the distance of closest approach and the subscript $p$ denotes the value for the field electron. ( $p$ plays the role of the collision parameter.)

$$
\left.\begin{array}{c}
C^{\prime \prime}=\frac{k_{p}}{k_{0}} \frac{\cos ^{2} \varphi_{p}}{\cos ^{2} \varphi_{m}(q)}, \\
k_{p}=\frac{\omega_{p}(p)}{v_{\|\|}(p)}=\left(\frac{n_{p}}{n_{0}}\right)^{1 / 2} \frac{\cos (\beta+\gamma)}{\cos \beta} k_{0}, \\
A=-\int_{0}^{p} \frac{2 \pi^{2} \epsilon^{2} \cos \varphi_{p}}{m k_{q} \cos \varphi_{m}(q)} \frac{\partial F(q)}{\partial v_{\|}} d q,
\end{array}\right\}
$$

with q measured from the field electron.

$$
\cos \varphi(q) \equiv \cos \varphi\left(p^{\prime}, p\right)=\frac{1}{p} \int_{0}^{\eta} \cos \varphi_{q} d q=\frac{1}{p-p^{\prime}} \int_{p^{\prime}}^{p} \cos \varphi_{p^{\prime}} d p^{\prime}
$$

The exponent $A$ can be rewritten

$$
A=-\int_{0}^{p} \frac{2 \pi e^{2} \cos \varphi_{p}}{m k_{p^{\prime}} \cos \varphi_{m}\left(p^{\prime}, p\right)} \frac{\partial F\left(p^{\prime}\right)}{\partial v_{\|}} d p^{\prime}
$$

$F\left(p^{\prime}\right)$ refers not only to the value at $p^{\prime}$ but also for $v_{\|}^{\prime}=v_{0} \cos \beta / \cos \left[\beta+\gamma\left(p^{\prime}\right)\right]$.

It should be noted that there is a reciprocity between emission and absorption. If the field electron, whose position of closest approach is given by $x,-y,-z$ and which is contributing 
to the damping of the test electron's wave at this point, has velocity 'II $_{\|}$. then another field electron with the same velocity at $x . y, z$ will have its wave damped by the test rectron at the origin. The test electron's wave has wavenumber changing from $k_{0}$ at $\mathbf{R}=0$ to $k_{p}$ at $x$, $-y,-z$, while the field electron at $x, y, z$ has wavenumber changing from $k_{p}$ locally to $k_{0}$ at the origin.

Taking the square of $\Delta v_{\|}$in Eq. (44), multiplying by the number of such encounters per unit time, namely $\left[F\left(p, v^{\prime} \|\right) d v_{\|}\right] u p d \varphi_{0} d p$, and averaging over a wavelength $2 \pi / k_{p}$ in $p$, the contribution to $\left\langle\left(\Delta v_{\|}\right)^{2}\right\rangle$ is

$$
\left[F d k_{\mu}\right] d \varphi_{u} d p \frac{4 \pi C^{\prime 2} e^{4} k_{y z}^{2} \cos ^{2} \beta}{m^{2} u k_{p}} e^{-2 A} .
$$

Making the substitutions $v_{\|}=v_{0} \cos \beta / \cos (\beta+\gamma)$ and $d v_{\|}=v_{\|} u d \beta / v_{0} \cos \beta$, the limits $v^{\prime} \|= \pm \infty$ give $3+\gamma= \pm \pi / 2$, and the diffusion coefficient is

$$
\begin{aligned}
\frac{1}{2}\left\langle\left(\Delta \nu^{\prime} \|\right)^{2}\right\rangle & =\frac{2 \pi \epsilon^{4}}{m^{2}} \int_{-\pi / 2}^{+\pi / 2} d \varphi_{0} \int_{0}^{x} d p \int_{-\pi / 2-\gamma}^{+\pi / 2-\gamma} d \beta C^{\prime 2} \frac{k_{y z}^{2} \cos ^{2} \beta}{k_{p}^{\prime} \cos (\beta+\gamma)} F\left(p, \frac{v_{0} \cos \beta}{\cos (\beta+\gamma)}\right) \\
& \times \exp \left[\int_{0}^{p} \frac{4 \pi^{2} e^{2} \cos \varphi_{p}}{m h_{p}^{\prime} \cos \varphi_{m}\left(p^{\prime}, p\right)} \frac{\partial F\left(p^{\prime}\right)}{\partial v_{\|}} d p^{\prime}\right] .
\end{aligned}
$$

Considering the special case where $F$ is everywhere Maxwellian,

$$
F(p)=n_{p}\left(\frac{m}{2 \pi T_{p}^{\prime}}\right)^{1 / 2} \exp \left[-\frac{m v_{0}^{2} \cos ^{2} \beta}{T_{p} \cos ^{2}\left(\beta+\gamma_{p}\right)}\right]
$$

and integrating $E_{q} .(48)$ by parts with respect to $p$ for this case

$$
\begin{aligned}
\frac{1}{2}\left\langle\left(\Delta v_{\|}\right)^{2}\right\rangle= & \frac{2 \pi n_{0} e^{4}}{m^{2}} \int_{-\pi / 2}^{+\pi / 2} d \varphi \cos ^{2} \varphi_{0}\left(\frac{k_{0}^{2} \lambda_{D}^{2}}{\pi v_{0}}\right)\left[\int_{-\pi / 2}^{+\pi / 2} d \beta C^{\prime 2} \cos \beta\right. \\
& \left.+\int_{0}^{\infty} d_{p} \int_{-\pi / 2}^{+\pi / 2} d \beta \cos \beta e^{\gamma} \frac{\partial}{\partial p}\left(C^{\prime 2} \frac{T_{p}}{T_{0}}\right)\right],
\end{aligned}
$$

Where $Y$ is the term in square brackets in Eq. (48) and in the limits for $\beta$ the approximation $(\pi / 2 \pm \gamma) \simeq \pi / 2$ has been used. $\lambda_{D}^{2}=T_{0} / 4 \pi n_{0} \epsilon^{2}$. 
From Eqs. (20) and (27) the factor $\left(^{\prime 2}\right.$ is given by

$$
C^{\prime 2}=\frac{1+2 \frac{\bar{d} k}{k_{0}}}{1+\frac{i_{k}}{k_{0}}}
$$

to first order in $\delta k$. If $n$ is linear in $x$ and $L_{s}$ is a constant then $\delta k$ is linear in $x$ and $C^{\prime \prime}$ is unity and $\partial C^{\prime \prime} / \partial_{p}=0$. Iir this case, with uniform temperature $\left(T_{p}=T_{0}\right)$, the second term in Eq. (49) is zero and, as will be seen below, the first term balances the $\left\langle\Delta v_{\| l}\right\rangle$ term in the Fokker-Planck operator. This is the correct physical property for uniform temperature. However, if $\delta k$ varies nonlinearly then $C^{\prime 2}$ differs by a small amount from unity and has a small derivative in $p$. This gives the non-physical result of energy transport in the presence of uniform temperature. This discrepancy must be due to the various approximations which have been made. In particular, there is a higher order WKB approximation to $k^{2}$ involving $C^{\prime}$ and its derivative. For these reasons (" is set equal to unity in Eq. (49),

With this modification and $T_{p}=T_{0}$ Eq. $(49)$ reduces to

$$
\frac{1}{2}\left\langle\left(\Delta v^{\prime} \|\right)^{2}\right\rangle=\frac{\pi n \epsilon^{4} v_{T}^{2}}{m^{2} v_{0}^{3}}
$$

and changing the notation from vo to $v^{\prime} \|$

$$
C_{W}(f)=\frac{2 \pi n \epsilon^{4}}{m^{2}} \frac{\partial}{\partial v_{\|}} \frac{1}{v_{\|}^{2}}\left(f+\frac{v_{T}^{2}}{2 v_{\|}} \frac{\partial f}{\partial v_{\|}}\right)
$$

which is zero for Maxwellian $f$. The resultant Fokker-Planck operator when there is a temperature gradient is

$$
C_{W}(f)=\frac{2 \pi n \epsilon^{4}}{m^{2}} \frac{\partial}{\partial v_{\|}}\left[\frac{v_{T}^{2}}{v_{\|}^{3}} \frac{\partial f}{\partial v^{\prime} \|} \int_{+\pi / 2}^{-\pi / 2} \frac{d \varphi_{0}}{\pi} \cos ^{2} \varphi_{0} \int_{-\pi / 2}^{+\pi / 2} d \beta \cos \beta \int_{0}^{\infty} d p e^{Y} \frac{\partial}{\partial p}\left(\frac{T_{p}}{T_{0}}\right)\right]
$$

$Y$ in this case is given by

$$
Y_{m}=-\int_{0}^{p} d p^{\prime} \frac{\pi^{1 / 2} \cos \varphi_{p}}{\lambda_{D}^{2} k_{p}^{\prime} \cos \varphi\left(p^{\prime}, p\right)}\left(\frac{v_{\|}^{\prime}}{v_{T_{0}}}\right)\left(\frac{n_{p}^{\prime}}{n_{0}}\right)\left(\frac{T_{0}}{T_{p}^{\prime}}\right)^{3 / 2} \exp \left(-\frac{m v_{\|}^{\prime 2}}{T_{p}}\right)
$$

with $v_{\|}^{\prime}=v_{0} \cos \beta / \cos \left(\beta+\gamma_{p}^{\prime}\right)$. 
For the general ase with non- Maxwellian tail to F, integrating by parts in Eq. (48)

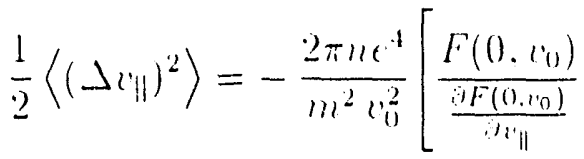

$$
\begin{aligned}
& \left.-\int_{-\pi / 2}^{+\pi / 2} \frac{d \varphi_{u}}{\pi} \cos ^{2} \varphi_{u} \int_{0}^{\infty} d p e \frac{\partial}{\partial p}\left[\int_{-\pi / 2}^{+\pi / 2} d \beta \frac{\cos ^{2} \beta F\left(p, v^{\prime} \|\right)}{\cos \left(\beta+\gamma_{p}\right) \frac{\partial F\left(p, u^{\prime} \|\right)}{\partial v_{\|}}}\right]\right] \text {. }
\end{aligned}
$$

The resultant Fokker-Planck equation for this case is given in the next section.

\section{Summary and Discussion}

The emission and absorption of electron plasma waves has been studied for the case where the magnetic shear is only moderate, so that the change in the electrostatic wake of a superthermal test electron can be treated as a perturbation from the uniform magnetic field case. Only slab geometry has been treated with the magnetic surfaces taken as planes normal to the $x$-axis. The part of the wake which, for uniform rnagnetic field, has spherical symmetry behind the test electron has been identified as the plasma wave emission of the electron. The dominant effect of the magnetic shear and this spherical symmetry is that at

the magnetic surface at distance $x$ from the test electron, the "resonant" electrons causing the Landau damping of the wave have the velocity

$$
v_{\|}^{\prime}=\frac{v_{\|}^{\prime} \cos \beta}{\cos (\beta+\gamma)}
$$

Here $v_{\|}$is the velocity of the test electron parallel to $\mathbf{B}(0), \beta$ is the angle between $\mathbf{B}(0)$ and the projection of $\mathrm{k}$ on the $y z$-plane at $x=0$ and $\gamma$ is the angle between $\mathbf{B}(x)$ and $\mathbf{B}(0)$.

Using the results of Eqs. (43) and (54) the Fokker-Planck equation for the rate of change of the electron distribution function due to the emission and absorption of electron plasma waves is 


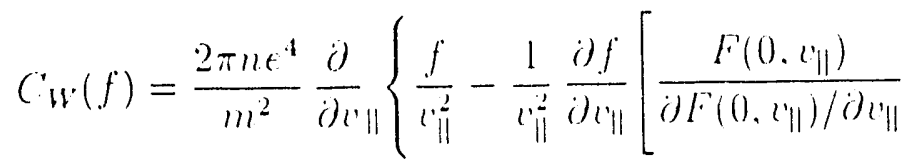

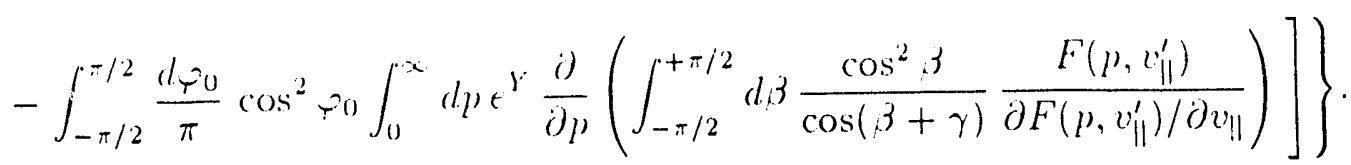

Here $Y$ is given by the exponent in square brackets in $\mathrm{Eq}_{1} .(48), v^{\prime}$ is given by $\mathrm{Eq}_{1} .(55), \varphi_{p}$ is the angle between $\mathrm{k}$ and the $y z$-plane at position $p$ and $p$ is measured along each ray. The integral to infinity in $\mu$ will involve reflections of the ray where $k_{p}$ has fallen to the value $k_{p}=k_{y z}=k_{u} \cos \varphi_{u}$. The coordinates of the point $p$ are $x=p \sin \varphi_{m}, y=-p \cos \varphi_{m} \sin \beta$, $z=-p \cos \varphi_{m} \cos \beta$ with

$$
\cos _{\varphi^{\prime} m}=p^{-1} \int_{0}^{p} \cos \varphi_{p} d p=p^{-1} \cos \varphi_{0} \int_{0}^{k}\left(k_{0} / k_{p}\right) d p
$$

and

$$
k_{p}=k_{0}\left(\frac{n_{p}}{n_{1}}\right)^{1 / 2} \frac{\cos (\beta+\gamma)}{\cos \beta} \text {. }
$$

$F$ is the one-dimensional distribution function $\int 2 \pi v_{\perp} f d v_{\perp}$.

For most applications, such as the transport of energy or momentum, when moments are taken of $C_{w}$ the integral with respect to $v_{\perp}$ will convert $f$ to $F$ in $\mathrm{Eq} .(56)$ and the first term in the square brackets will cancel the $f / v_{\|}^{2}$ term, leaving only the second term in the square brackets. When $f$ is Maxwellian $\left(f_{m}\right)$ for all $v^{\prime}$ and is a function of only the single spatial coordinate $x$, the form of the net operator is given by Eq. (52), namely

$$
C_{w}\left(f_{m}\right)=\frac{4 \pi n \epsilon^{4}}{m^{2}} \frac{\partial}{\partial v} \|\left[\frac{f_{m}}{v_{\|}^{2}} \int_{-\pi / 2}^{+\pi / 2} \frac{d_{\gamma_{0}} \cos ^{2} \varphi_{0}}{\pi} \int_{-\pi / 2}^{+\pi / 2} d \beta \cos \beta \int_{0}^{\infty} d p e^{\gamma} \frac{\partial}{\partial p}\left(\frac{T_{p}}{T_{0}}\right)\right]
$$

with $Y$ given by Eq. (53).

The importance of magnetic shear and the spherical symmetry of each wake can be 
illustrated by considering an approximate form for the damping exponent in Eq. (53)

$$
\begin{aligned}
& Y \simeq-\mu_{0} \int_{0}^{\prime \prime}(1)^{\prime} \exp \left[x_{1}^{2}-\frac{x_{0}^{2}}{\left(1-\frac{p^{\prime} \sin +\tan 3^{3}}{L_{s}}\right)^{2}\left(1-\frac{L^{\prime} \sin \hat{L}}{L_{T}}\right)}\right] \\
& \simeq \mu_{0} \int_{0}^{p} d p^{\prime}(x)\left[-u_{0}^{2} \mu^{\prime} \sin p\left(\frac{2 \tan 3}{L_{s}}+\frac{1}{L_{T}}\right)\right]
\end{aligned}
$$

for small $p$. Here $\mu_{0}$ is the value of the integrand in $\mathrm{Eq}_{\mathrm{q}}$. (53) at $p=0$ and a linear decrease in $T_{e}$ has been taken as an example. For $|\tan \beta|<L_{T} / 2 L_{s}$ the situation is similar to that treated by Rosenbluth and Liu and $Y$ saturates for $p \sin \varphi>L_{T} / x_{0}^{2}$. With $\mu_{0}^{-1}$ greater than $L_{T} / x_{0}^{2}$, that is $x_{0}>x_{m}$ from $\mathrm{Eq} .(16)$, most of the wave energy is reflected and is reabsorbed in the central part of the plasma. For $\tan \xi \gtrsim L_{T} / 2 L_{s}$ the value of $p$ for saturation is reduced and $x_{m}$ is reduced. But for $-\tan ;>L_{T} / 2 L_{s}$ the effective mean free path for the wave is limited to approximately $L_{s} / 2 x_{0}^{2}$ tan 3 because the positive exponent in Eq. (60) causes a rapid decrease in the local damping length for $p$ greater than this value. Provided $\sin \varphi$ is not too small, the wave energy is absorbed before reflection occurs and there is increased energy transport. The value of $x_{m}$ is increased.

In the work of Rosenbluth and Liu a formula was derived for the change of electron energy due to the emission and absorption of the plasma waves. Using the form of $C_{W}$ given in Eq. (42), the corresponding rate of change of electron energy is

$$
\frac{\partial \mathcal{E}}{\partial t}=\int d^{3} v \cdot \frac{m v^{2}}{2} C_{w}(f)=2 \int_{0}^{\infty} d v_{\|} m v_{\|}\left(F\left\langle\Delta v_{\|}\right\rangle-\frac{1}{2}\left\langle\left(\Delta v_{\|}\right)\right\rangle^{2} \frac{\partial F}{\partial v_{\|}}\right) .
$$

For the case where $F$ is Maxwellian, the shear is negligibly small $(\gamma \rightarrow 0)$ and each ray has constant direction $\left(\varphi_{p}=\right.$ constant) $\mathrm{Eq}_{\mathrm{q}}$ ( $(61)$ reduces to

$$
\frac{\partial \mathcal{E}}{\partial t}=-\frac{4 \pi n e^{4}}{m} \int_{0}^{\infty} d v_{\|}\left[\frac{F}{v^{\prime} \|}+\frac{1}{v_{\|}^{2}} \frac{\partial F}{\partial v_{\|}} \frac{1}{2 \pi m} \int d \Omega \frac{k_{\|}}{k} \int_{0}^{\infty} d_{\mu} T_{p} e^{-\mu}\right]
$$

where $d \Omega$ is the element of solid angle $\cos \varphi d z d \varphi$ and $\mu$ is given by

$$
\mu=\int_{0}^{p} d p^{\prime} \frac{\pi^{1 / 2} r_{0}}{k_{p^{\prime}} \lambda_{D}^{2}}\left(\frac{n_{p^{\prime}}}{n_{0}}\right)\left(\frac{T_{0}}{T_{p^{\prime}}}\right)^{3 / 2} \exp \left(-\frac{m v_{\|}^{2}}{T_{p^{\prime}}}\right) .
$$


The corresponding formula obtained by Rosenbluth and Lin' from their Eqs. (14)-(16) is

$$
\left(\frac{\partial \mathcal{E}}{\partial t}\right)_{\mathrm{RL}}=-\frac{1}{(2 \pi)^{3}} \int d^{3} \mathrm{k}\left|\mu_{\partial x}\right||\lambda|\left[T_{0}-\int_{0}^{\infty} d \mu T_{i}, t^{-\mu}\right]
$$

where

$$
v_{y x}=\omega_{p} k_{\|} k_{x} / h^{3}
$$

and

$$
\lambda=\left(\frac{\pi}{2}\right)^{1 / 2} \frac{1}{k_{x} k \lambda_{D}^{3}} \exp \left(-\frac{1}{2 k^{2} \lambda_{D}^{2}}\right) .
$$

Making the substitution $k \lambda_{D}=1 / \sqrt{2} x_{0}$ this expression reduces to

$$
\left(\frac{\partial E}{\partial t}\right)_{\mathrm{RL}}=-\frac{4 \pi n e^{4}}{m} \int_{0}^{\infty} d v_{\|}\left[\frac{F}{v_{\|}}+\frac{1}{v_{\|}^{\prime}} \frac{\partial F}{\partial v_{\|}} \frac{1}{2 \pi m} \int d \Omega \frac{k_{\|}}{k} \int_{0}^{\infty} d \mu T_{p} e^{-\mu}\right] .
$$

Equation (62) is identical with Eq. (64).

\section{Acknowledgments}

This work was supported by the U.S. Department of Energy contracts \#DE-FG05-80ET53088 and \#DE-F(105-88ER-53266. 


\section{Appendix}

The change of phatse is recunired for an increase $\delta r$ in $r \equiv\left(y^{2}+z^{2}\right)^{1 / 2}$. If the ray passing

through the point $r, r$ has components of $\mathrm{k}$ at $R=0$ given by $k_{r}=k_{y z}=k_{0} \cos \varphi u$, $k_{x}=k_{0} \sin \varphi_{0}$, the phase at the point $x, r$ is

$$
\Theta=\int_{0}^{R \cos \varphi^{\prime \prime n}} k_{0} \cos \varphi_{0} d r+\int_{0}^{R \sin \nu_{m}}\left[k_{0} \sin \varphi_{0}+\delta k_{x}(x)\right] d x
$$

The corresponding phase at $x, r+\delta r$, where the initial angle of the ray has changed to po- $\delta \varphi_{0}$. is

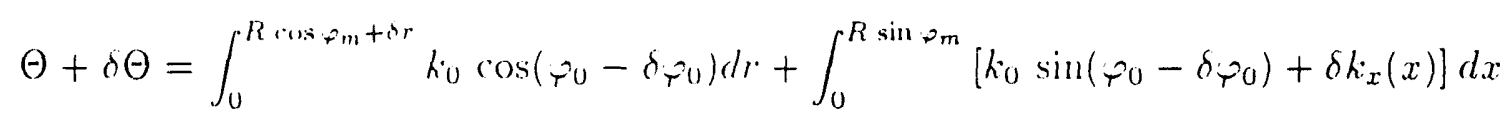

or using Eq. (Al)

$$
\begin{aligned}
\delta \Theta & =k_{y z} \delta r+k_{0} R \delta_{\varphi}\left(\sin \varphi_{0} \cos \varphi_{m}-\cos \varphi_{0} \sin \varphi_{m}\right) \\
& =k_{y z}\left[\delta r+R \delta_{\varphi_{m}} \frac{\partial_{\varphi_{u}}}{\partial \varphi_{m}}\left(\tan \varphi_{0} \cos \varphi_{m}-\sin \varphi_{m}\right)\right] .
\end{aligned}
$$

From $E$ l. $(23) \cos \varphi_{0}=\cos \varphi_{m} /\left(1-\frac{\bar{\delta}_{k}}{\bar{k}_{0}}\right)$ and hence $\sin \varphi_{0}=\sin \varphi_{m}\left(1-\frac{\bar{\delta}_{k}}{k_{0}} \cot ^{2} \varphi_{m}\right)$. Also $R \delta \varphi_{m}=\delta r \sin \varphi_{m}$. Hence

$$
\delta \Theta=k_{y z} \delta r\left[1-\frac{\partial \varphi_{0}}{\partial \varphi_{m}} \frac{\bar{\delta} k}{k_{0}}\right] \simeq k_{y=} \delta r\left[1-\frac{\bar{\delta} k}{k_{0}}\right]=k_{0} \delta r \cos \varphi_{m}
$$

since to zero order in $\delta k / k, \partial \varphi_{0} / \partial \varphi_{m}=1$. 


\section{References}

1. A.A. Ware and J.('. Wiley, IF' Report \#,561, July, 199!2. to be published.

2. V.K. Decyk. (i.J. Morales. J.M. Dawson, and H. Abe. 13th European ('onf. Contr. Fusion, Pt. II, p. 358, 1986.

3. N. Rostoker and M.N. Rosenbluth, Phys. Fluids 3, 1 (1960).

4. V.K. Decyk, Phys. Fluids 25, 1205 (1982).

5. P. Amendt and N. Rostoker, Phys. Rev. A 29, 1326 (1984).

6. M.N. Rosenbluth and (C.S. Liu, Phys. Fluids 19, 815 (1976).

7. M.N. Rosenbluth and N. Rostoker, General Atomic Report GAMD-663, Pt. III, p. 54, Eq. (72), 1959.

8. T. O’Neil, Phys. Rev. Lett. 55, 943 (1985). 


\section{Figure Captions}

1. This figure represents a magnet ic surface at the distance $x$ from the test electron. The line maked $"$ is the muperturbed orbit of a field electron with velocity a relative to the test electron. P is the print of elosest approach.

2. The two functions $\int_{-\infty}^{r} \cos \left(k l^{2} / 2 R\right) d l$ and $\int_{-\infty}^{t} \sin \left(k \ell^{2} / 2 R\right) d l$ are plotted as functions of $\ell$. 


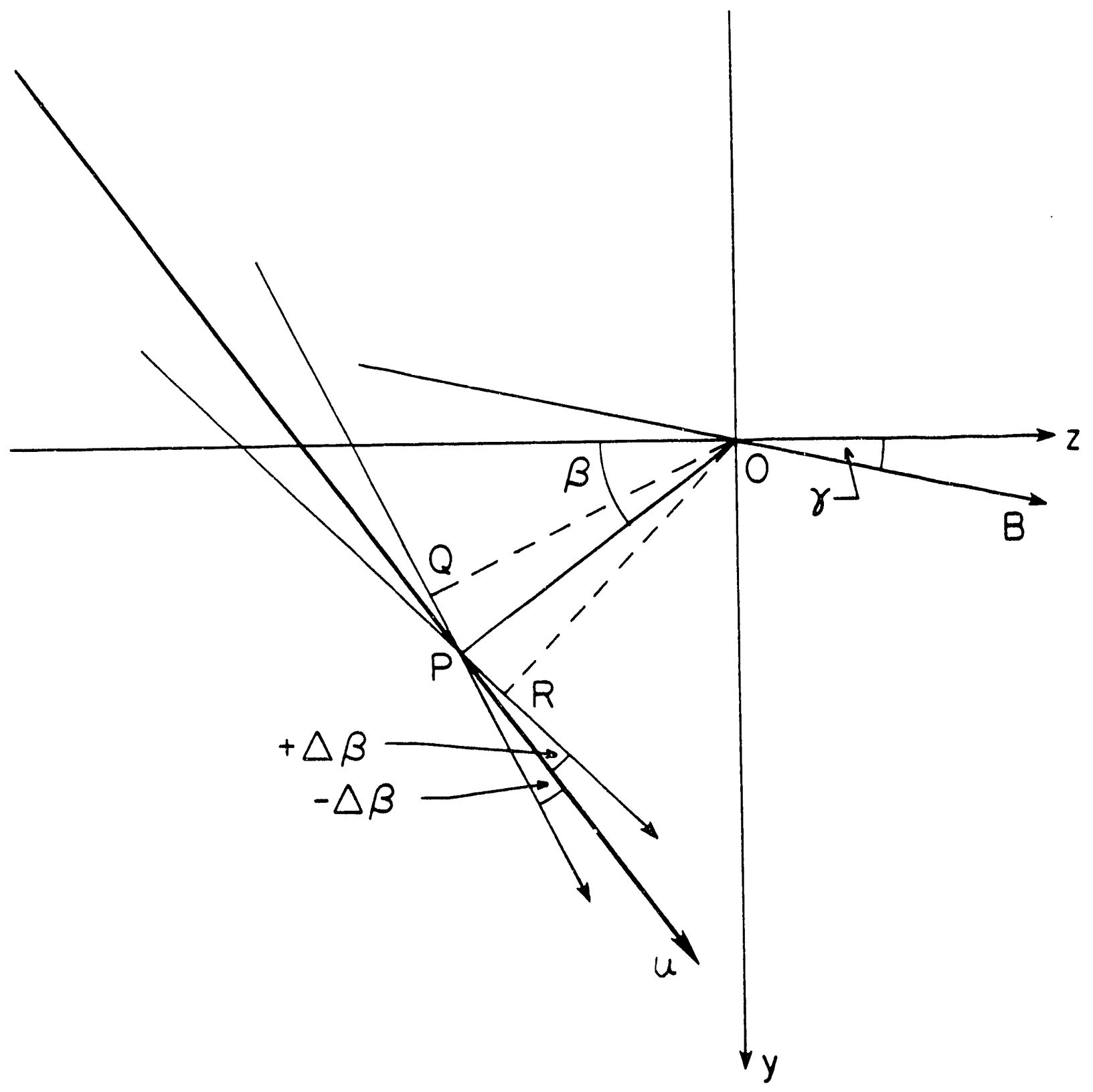

Fig. 1 


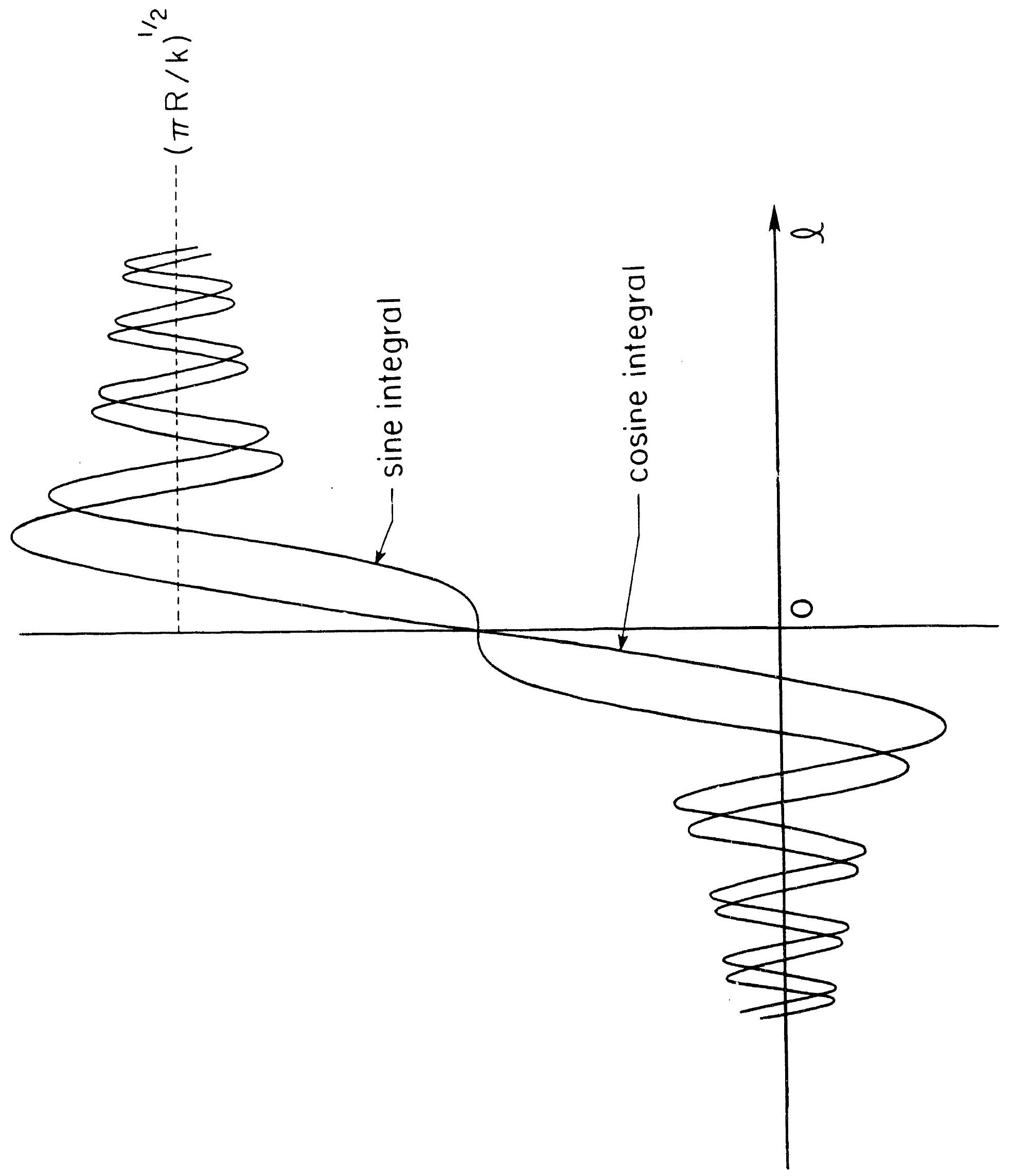

Fig. 2 

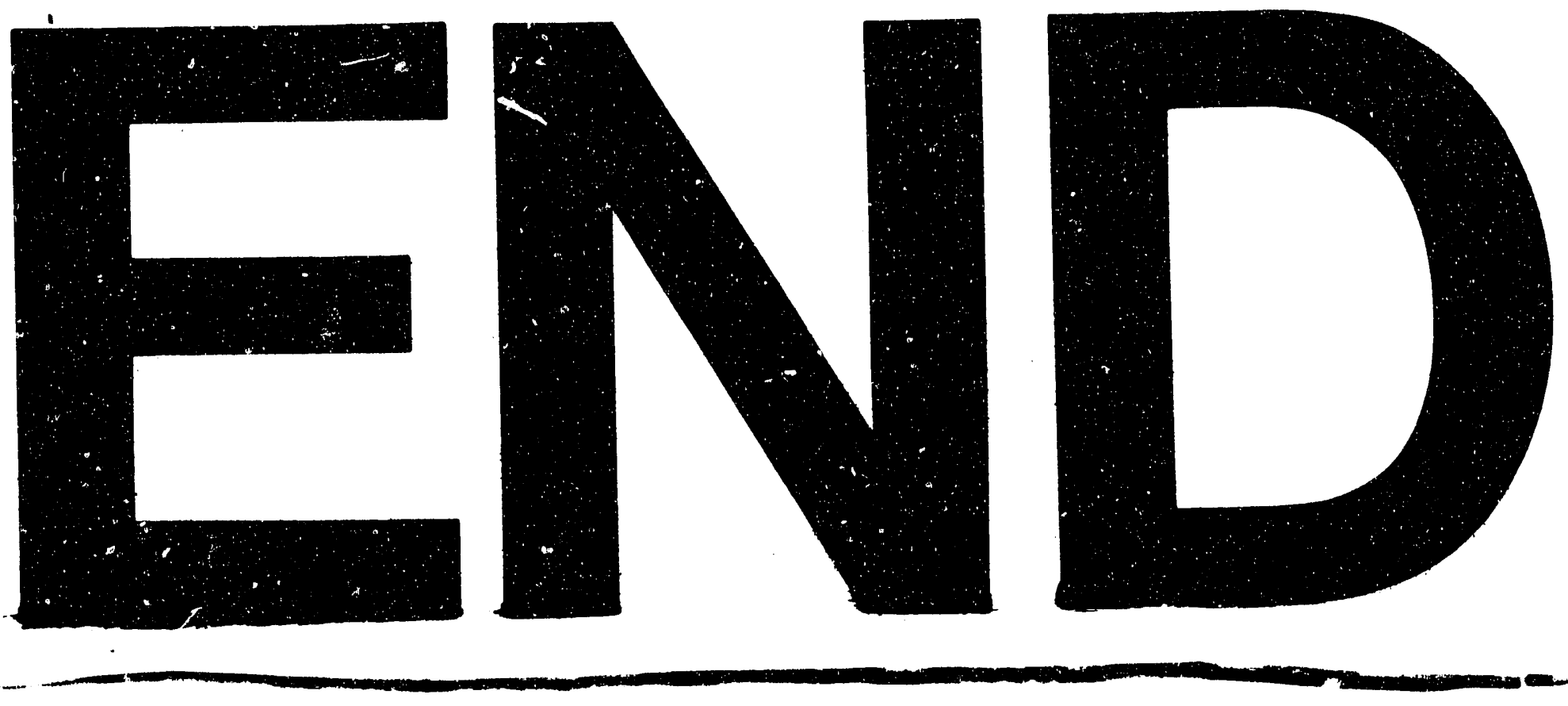

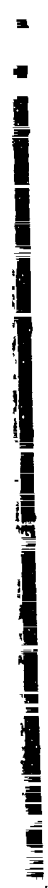
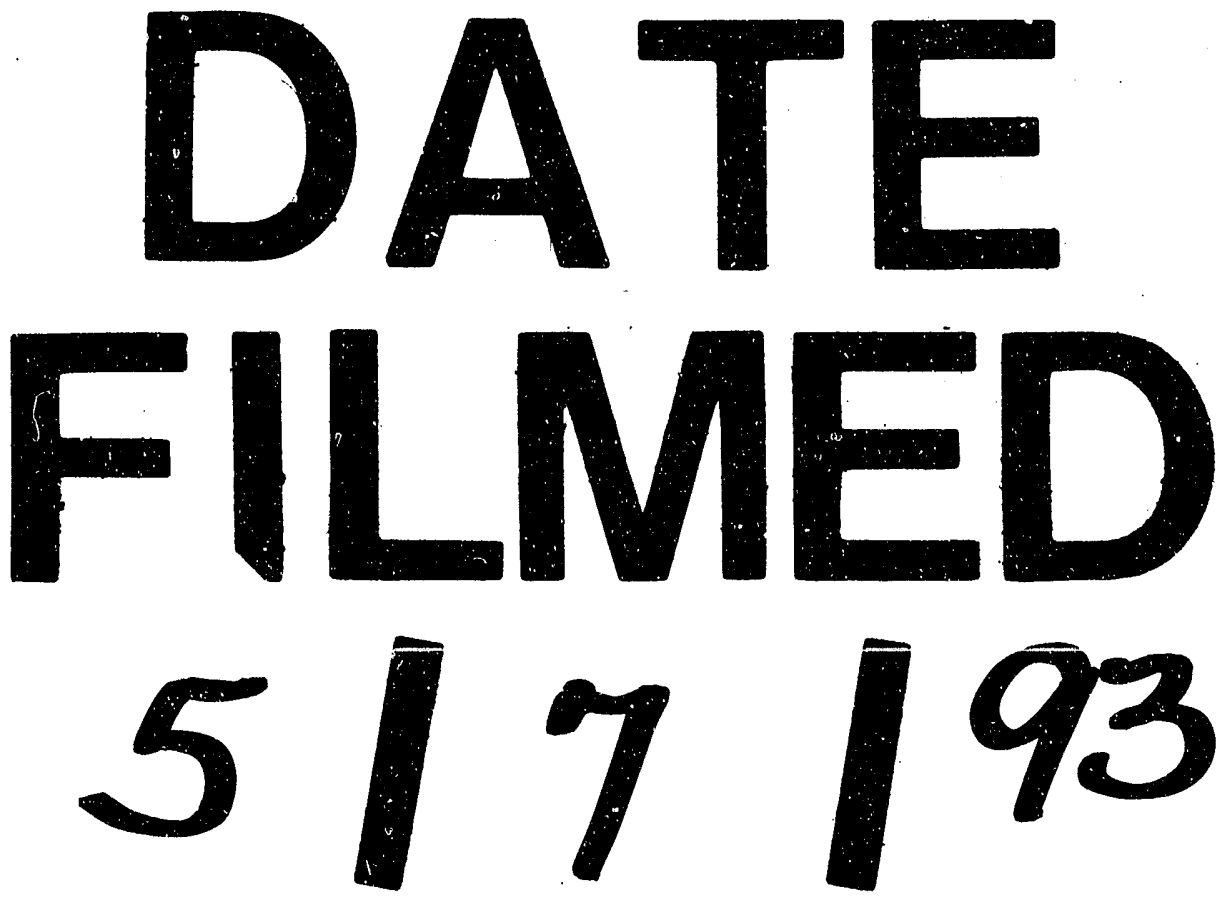
| 